\title{
Effect of Relative Movement between the Shroud and Blade on Tip Leakage Flow Characteristics
}

\author{
Xiaochun Wang, Jianhua Wang *, Fei He * and Hong Zhang \\ Department of Thermal Science and Energy Engineering, University of Science and Technology of China, \\ Jinzhai Road 96, Hefei 230027, China; sprdawn@usts.edu.cn (X.W.); kingsisi@mail.ustc.edu.cn (H.Z.) \\ * Correspondence: jhwang@ustc.edu.cn (J.W.); hefeihe@ustc.edu.cn (F.H.); Tel.: +86-551-63600945 (J.W. \& F.H.) \\ Academic Editor: Fernando Porté-Agel \\ Received: 22 August 2017; Accepted: 9 October 2017; Published: 13 October 2017
}

\begin{abstract}
An experimental and numerical investigation into the tip leakage flow of a turbine rotor is carried out using a particle image velocimetry (PIV) system and the commercial software ANSYS CFX 14.0. The specimen used in this work is a typical GE- $E^{3}$ model with a new squealer tip design. The experimental data are used to create a turbulence model and numerical strategy. Through the validated turbulence model and numerical strategy, simulations are carried out to compare the characteristics of the tip leakage flow in three cases: (1) the blade is rotating, but the shroud is stationary, which is the real status of turbine rotor operation; (2) the blade is stationary, but the shroud moves, to simulate their relative movement; (3) the blade is stationary, and the shroud is also stationary, this is a simplified case, but has been widely used in the experiments on rotor tip leakage flow. Detailed analysis of the flow phenomena shows that the second case is a reasonable alternative approach to simulate the real state. However, the flow patterns in the third case exhibit some evident differences from the real status. These differences are caused by the inaccurate viscous force arising from the stationary blade and shroud. In this work, a modification method for the experiments conducted in the third case is firstly proposed, which is realized through adding an imaginary roughness at the shroud wall to be close to the real viscous effect, and to thereby reduce the deviation of the experiment from the real case. According to the results calculated by ANSYS CFX, the flow structure in the modification case is very close to the real status. Besides, this modification case is an easy and cheap way to simulate the real tip leakage flow.
\end{abstract}

Keywords: tip leakage flow; numerical simulation; wall roughness; particle image velocimetry; relative movement

\section{Introduction}

Increasing the efficiency and specific power output of turbines are two major goals in gas turbine designs. These goals can be achieved by increasing the turbine entry temperature. Therefore, modern gas turbines are operating at a temperature far higher than the current blade materials could afford. The blade's tip is one of the regions with the highest heat transfer rate in the entire rotor blade, because the leakage flow driven by the large pressure difference between pressure side and the suction side is accelerated in the tip gap region and impinges on the tip. Bunker et al. [1] provided a review of the previous experimental and computational investigations into the tip leakage, and pointed out that the tip surface is exposed in the most extreme aero-thermal environment within gas turbines. Kwak et al. [2] reported that the overall heat transfer coefficient on the blade tip showed a higher value than that on the shroud and in the near tip regions of the blade pressure and suction sides, through their experiment. Therefore it is of great importance to study the performance of fluid flow and heat transfer in the tip leakage and to analyze the effect of leakage flow characteristics on the turbine blade and mainstream. 
The experimental investigations on the fluid flow and heat transfer in the tip gap region of the turbine rotors are usually quite difficult. As shown in Figure 1, the experiments were conducted in three typical methods in the open literature: (1) Most experiments were conducted under the conditions of BSSS (the blade is stationary, and the shroud is also stationary) [2-6]; (2) To simulate blade rotation, some experiments were conducted under a relative movement condition of BSSM (the blade is stationary, but the shroud moves along a straight line, to simulate relative movement) [7-11]; (3) Few experiments were performed with BRSS (the blade is rotating, but the shroud is stationary, which is the real status of turbine rotor operation) [12-17]. Obviously the third kind of experiment condition, BRSS, is a real condition of a turbine rotor operating, but it is very expensive to create the real experimental environment of a gas turbine, and therefore BRSS experiments were nearly all carried out at low rotational speeds. Yang et al. [18] used the numerical approach to study the rotation effects on the tip leakage flow and heat transfer, and they found that the relative motion of the shroud significantly increases the averaged heat transfer coefficient on the blade tip. Thus there is no guarantee of the accuracy of the results obtained in experiments conducted under the conditions of BSSS, BSSM and BRSS with a low rotational speed. However there are a few investigations into the systematic and comprehensive analysis and comparison of the tip leakage flow characteristics under different experiment methods so far. Acharya et al. [19] numerically investigated the effects of film cooling, heat transfer and aerodynamics on the blade tip and pressure and suction sides under the three experiment conditions, but their numerical simulations were carried out only at two low rotation speeds of 375 and $750 \mathrm{r} / \mathrm{min}$. Krishnababu et al. [20] compared the streamlines across the tip and contours of the heat transfer coefficient on the tip under BSSS and BSSM conditions through simulations without the consideration of film cooling.

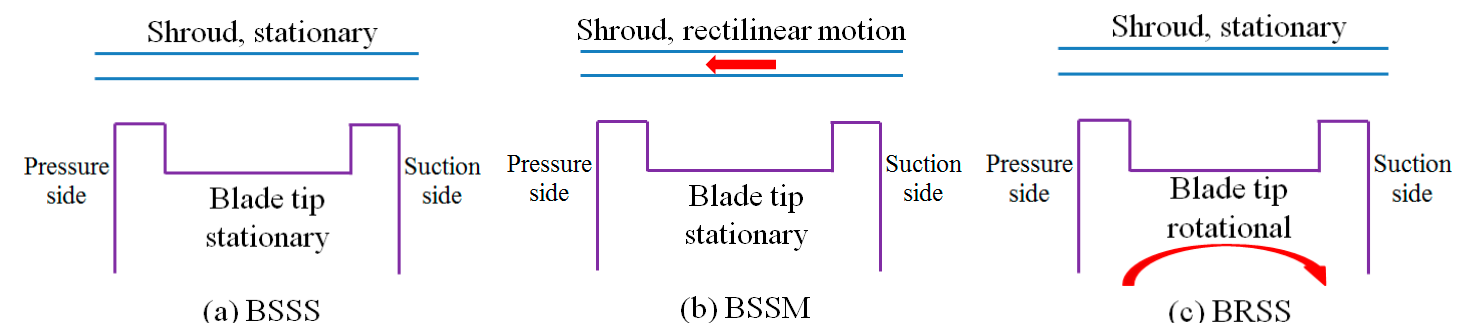

(a) BSSS

(b) BSSM

(c) BRSS

Figure 1. Three simulation models: (a) BSSS (blade is stationary and shroud is also stationary); (b) BSSM (blade is stationary, but shroud moves along a straight line); (c) BRSS (blade is rotating, but shroud is stationary).

To reduce the heat impinging of the leakage flow on the tip, the new rotor of the squealer tip with a grooved structure has been widely employed in the blades instead of the traditional flat tip. The groove acts as a labyrinth seal to increase the flow resistance, and the leakage mass flow is thereby decreased. Recently a great number of investigations have been conducted on the improvement and transformation of the squealer tip [21-25].

As for the simulation method, the large eddy simulation (LES) is deemed to be able to provide more accurate predictions of flow characteristics. Hahn et al. [26] used LES to investigate a fully three-dimensional swept-wing geometry featuring separation from a curved leading edge. Hahn et al. [27] present a systematic numerical investigation of different implicit large eddy simulations for massively separated flows. However the calculations by LES are time-consuming processes. In consideration of the complex blade geometry and huge computational grid scale in this research, Reynolds-averaged Navier-Stokes equations were used to solve the tip leakage flow.

The turbulence model and simulation method is one of the critical factors in the numerical simulation of tip leakage flow. Leschziner et al. [28] gave a detailed review of turbulence models in aeronautics. There are various turbulence models employed in the existing literatures, such as the standard k- $\omega$ model by Yang et al. [18], the SST k- $\omega$ model by Krishnababu et al. [20] and the RNG 
$\mathrm{k}-\varepsilon$ model by Yang et al. [29]. To validate the numerical strategy including the turbulence model, an experiment was performed in this work. Through the comparison between the experimental data and the numerical results obtained by different turbulence models, it was found that the RNG k- $\varepsilon$ turbulence model matched best and thereby the turbulence model and simulation method used are reasonable. As the open literatures indicate, most experiments were conducted in the simplified BSSS case, because the experimental expenses of BSSS are much lower than those of BRSS and BSSM. However, the deviation caused by the simplification and how to reduce the deviation were not considered up to now. Aiming at exhibiting the differences between the three experimental methods, and searching for a modification approach to the experimental data obtained by the simplified BSSS method, this work experimentally and numerically investigated the tip leakage flow of a turbine rotor. A three-bladed stationary linear cascade was designed and manufactured. The corresponding flow behavior of the tip leakage, mainstream and cooling air were captured by a particle image velocimetry (PIV) system. Using the validated numerical strategy, three experimental methods (BSSS, BSSM and BRSS) were simulated and are discussed at three rotation rates $(1000,2500$ and $4000 \mathrm{r} / \mathrm{min})$. Through the discussion, the flow characteristics of the tip leakage in BSSM case was found to be very close to the real state BRSS, and the differences of tip leakage flow between BSSS and BRSS were quantitatively evaluated by the tip leakage mass flow. Accordingly, a method based on correcting the wall viscous shear stress via shroud wall roughness is proposed to reduce the difference caused in BSSS case from the real case, BRSS.

\section{Experimental Apparatus and Procedures}

\subsection{Blade Model Geometry}

The tested blade model had the same profile as a GE- $\mathrm{E}^{3}$ rotor, but was scaled up three times to improve optical access and meet the requirements of PIV measurement. The geometry parameters of the blade are summarized in Table 1. There were two rows of cylindrical film cooling holes on the blade tip, the diameter of the film holes was $1.5 \mathrm{~mm}$, and the injection direction was perpendicular to the tip surface, as shown in Figure 2. The squealer at the suction side was along the blade profile, but at the pressure side there was a small gap with a size of $7.36 \mathrm{~mm}$ from the trailing edge, in order to decrease the accumulation of cooling air on the tip. On the pressure side of the blade tip there were five film holes outside the squealer, so that the cooling air injected from the film holes could restrain the leakage flow into the tip gap. Along the camber line of the tip, there were $11 \mathrm{film}$ holes to hinder the fluid passing through the suction side. The distance between adjacent holes was $2.5 \mathrm{~mm}$. The tested blade was made of stainless steel, and to avoid optical reflection, its surface was processed to approach a black body.

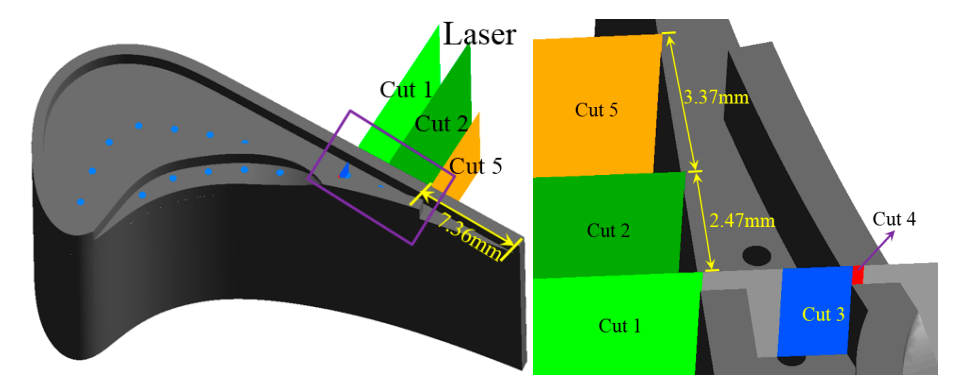

Figure 2. Blade model geometry illustration, and locations of PIV capture and simulation. 
Table 1. Tested blade parameters (scaled up by three times) and experimental conditions.

\begin{tabular}{cccc}
\hline Blade chord & $100 \mathrm{~mm}$ & \multicolumn{1}{c}{ Blade pitch } & $87 \mathrm{~mm}$ \\
Squealer depth & $4.5 \mathrm{~mm}$ & Blade height & $60 \mathrm{~mm}$ \\
Squealer rim thickness & $2.4 \mathrm{~mm}$ & Blade thickness & $24 \mathrm{~mm}$ \\
Inlet flow angle & $32 \mathrm{deg}$. & Blade exit angle & $24 \mathrm{deg}$. \\
\hline Average velocity of mainstream & \multicolumn{2}{c}{$0.1 \mathrm{~m} / \mathrm{s}$} \\
Average velocity of coolant flow at hole exits & \multicolumn{3}{c}{$0.3 \mathrm{~m} / \mathrm{s}$} \\
Reynolds number based on blade chord & \multicolumn{2}{c}{$1.3 \times 10^{4}$} \\
\hline
\end{tabular}

\subsection{Experimental System}

The experiment was performed in a three-bladed stationary linear cascade, which consisted of one fully profiled and two semi-profiled blade models. A schematic illustration of the test section is shown in Figure 3, and the experimental conditions are summarized in Table 1. The inlet section, test section and exit section were made of Plexiglas with a thickness of $8 \mathrm{~mm}$ to ensure good transparency. The mainstream region was extended upwards and downwards from the leading edge and trailing edge, respectively.

Filtered water was used as a mainstream and coolant, and injected from a reservoir by a diving pump. The inlet velocities of the mainstream and coolant were controlled by two valves and two turbine flow meters. To assure spatially uniform and undisturbed inlet flow, a square-hole honeycomb with $65 \%$ porosity and $40 \mathrm{~mm}$ length was set into the developing zone. The discharged water flowed back into the reservoir to recycle.

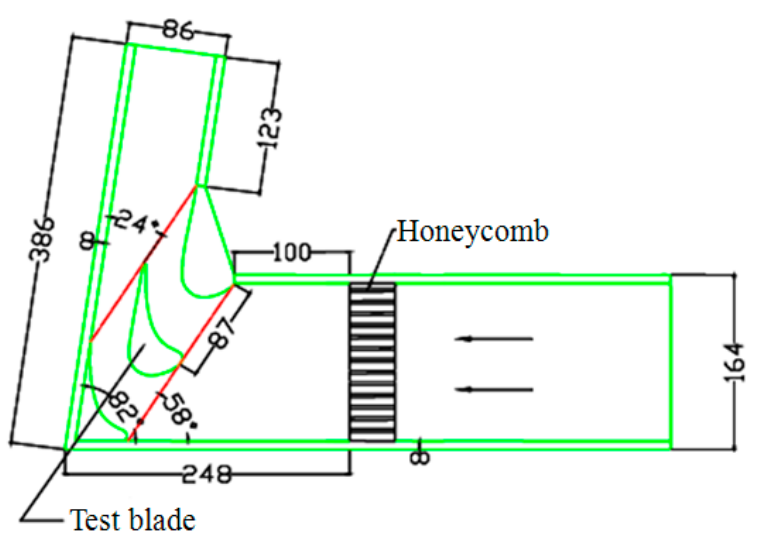

(a)

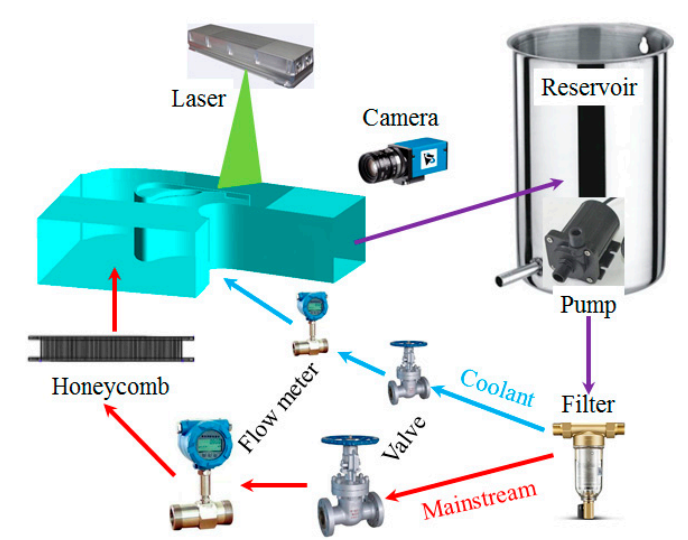

(b)

Figure 3. The schematic illustrations: (a) test section; (b) experimental apparatus.

\subsection{Particle Image Velocimetry Measurements}

The experiments were carried out at ambient temperature and pressure. The characteristics of the tip leakage flow were captured at two cross sections (cuts one and two) by PIV, which are marked in Figure 2. The tracer particles used in the PIV measurement were hollow glass spheres with an average diameter of $10 \mu \mathrm{m}$, and the density ratio of the spheres to water was 1.05. The illumination was provided by a double-cavity Gemini Nd:Yag pulsed laser, and its pulse energy was $200 \mathrm{~mJ} /$ pulse. The pulse delay between two laser pulses was set to $3 \mathrm{~ms}$ in this experiment. A HiSense $1024 \times 768$ pixels CCD camera was perpendicularly positioned to the light sheet. The size of an interrogation area was $32 \times 32$ pixels with $50 \%$ overlap. The images captured by the camera were processed into vector maps by the software Dantec Flowmap 1500. 


\subsection{Measurement Uncertainty}

The time-mean velocities at each point in a measured plane were calculated by a sequence of 300 instantaneous PIV vector maps in this experiment. For each velocity measurement, the bias uncertainty was caused by the camera calibration $\left(M=L_{0} / L_{1}\right)$, time interval of recording $(\Delta t)$ and particle displacement vector $(\Delta s)$ detection. Here $L_{0}$ is the size of the real object plane (in units of $\mathrm{mm}$ ), and $L_{1}$ means the pixel number in digital images.

In this experiment, the bias uncertainties were $L_{0}, L_{1}$, and $\Delta t$ are $0.0001 \mathrm{~m}, 0.15$ pixels, and $0.0000001 \mathrm{~s}$, respectively. According to error transfer formula, the absolute values of relative errors of the mean velocities were less than $2 \%$.

\section{Theoretical Analysis and Numerical Approach}

The fluid flow behavior of mainstream, leakage and cooling air in the gas turbine rotor can be described by compressible Reynolds-averaged Navier-Stokes (RANS) equations:

$$
\left\{\begin{array}{l}
\frac{\partial \rho}{\partial t}+\frac{\partial\left(\rho \bar{u}_{i}\right)}{\partial x_{i}}=0 \\
\frac{\partial\left(\rho \bar{u}_{i}\right)}{\partial t}+\frac{\partial\left(\rho \bar{u}_{i} \bar{u}_{j}\right)}{\partial x_{j}}=-\frac{\partial \bar{p}}{\partial x_{i}}+\frac{\partial}{\partial x_{j}}\left[\mu\left(\frac{\partial \bar{u}_{i}}{\partial x_{j}}+\frac{\partial \bar{u}_{j}}{\partial x_{i}}-\frac{2}{3} \delta_{i j} \partial \frac{\partial \bar{u}_{l}}{\partial x_{l}}\right)\right]+\frac{\partial\left(-\rho \overline{u_{i}^{\prime} u_{j}^{\prime}}\right)}{\partial x_{j}}
\end{array}\right.
$$

where Kronecker delta $\delta_{i j}=1$ if $i=j$ and $\delta_{i j}=0$ if $i \neq j$. In the experiments, water was employed, which can be regarded as an incompressible fluid, therefore, the equations can be written in a more concise form:

$$
\left\{\begin{array}{l}
\frac{\partial \bar{u}_{i}}{\partial x_{i}}=0 \\
\frac{\partial \bar{u}_{i}}{\partial t}+\frac{\partial\left(\bar{u}_{i} \bar{u}_{j}\right)}{\partial x_{j}}=-\frac{1}{\rho} \frac{\partial \bar{p}}{\partial x_{i}}+\frac{\partial}{\partial x_{j}}\left(v \frac{\partial \bar{u}_{i}}{\partial x_{j}}-\overline{u_{i}^{\prime} u_{j}^{\prime}}\right)
\end{array}\right.
$$

The commercial CFD software ANSYS CFX 14.0 was employed to solve the mathematical model described by the above equations. The entire computational domain had the same geometric structure and size as the experimental section shown in Figure 3, and was discretized with a finite control volume method. Using the commercial grid generation program ANSYS ICEMCFD 14.0, the mainstream region was filled with hexahedral mesh, while the tip region was filled with tetrahedral mesh. The hybrid grid consisted of both prisms and tetrahedral cells were used around the solid boundaries. The grid spacing was refined, so that the maximal value of the dimensionless distance, $y_{+}$, was less than one for the first nodes away from walls. Figure 4 shows the meshes assigned in the computation domain.

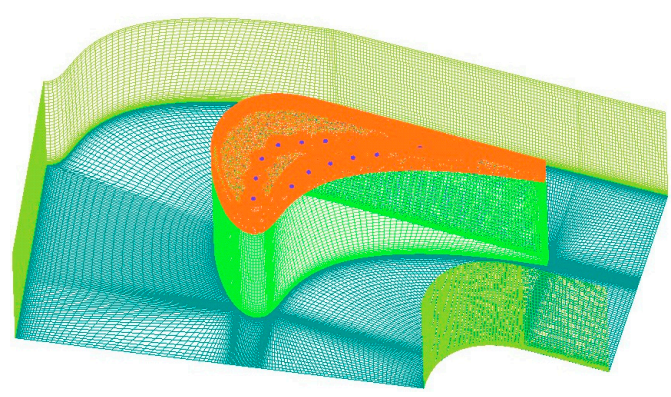

(a)

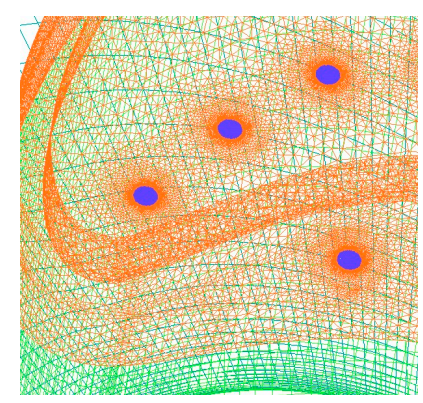

(b)

Figure 4. Computational meshes: (a) Meshes of the blade passage; (b) Meshes at the tip.

Simulations were conducted by RNG k- $\varepsilon$ turbulence model under the same conditions as the experiment. The wall function was set to be scalable. Water was chosen as the fluid material. The boundary conditions at all walls of the passage (including shroud, hub and the blade surfaces) were set to be no slip wall.

The grid independence of the numerical results was validated by testing five different meshes, as shown in Table 2. The numerical results of the tip leakage mass flow rate were compared, and finally 
the mesh with 5.96 million cells was employed in the following numerical simulations. The max relative deviation of the tip leakage mass flow was less than $0.13 \%$. The convergence of the simulations was judged on residual histories, and the convergence criterion was $10^{-5}$. This convergence criterion ensured that the average numerical uncertainty of velocities was less than $0.1 \%$.

Table 2. Validation of grid independence.

\begin{tabular}{lccccc}
\hline Mesh number (million) & 3.6 & 4 & 5.96 & 6.6 & 7.5 \\
\hline Tip leakage mass flow rate (kg/s) & 0.156690 & 0.156085 & 0.154668 & 0.154828 & 0.154729 \\
\hline
\end{tabular}

\section{Using Experimental Data to Validate Numerical Strategy}

Among the five cross sections shown in Figure 2, Cuts 1 and 2 were chosen to observe and investigate the tip leakage vortex in the tip gap. These cuts are through the centers of the first two holes near the trailing edge and perpendicular to the suction side surface. Figure 5 shows the mean velocities captured by PIV in the two cuts, and the corresponding simulated results. Comparing the locations of the vortex core, one can find that the numerical results obtained by the RNG k- $\varepsilon$ model match the experimental data well. Therefore, this numerical strategy can be used to discuss the differences of the three experimental conditions-BSSS, BSSM and BRSS—in the following simulations.

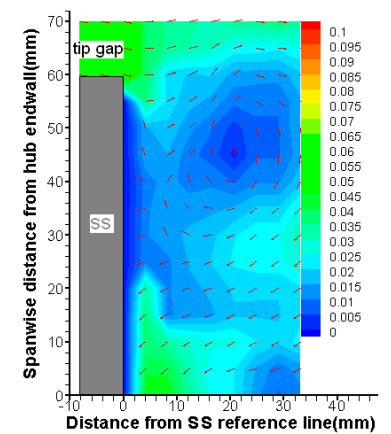

(a)

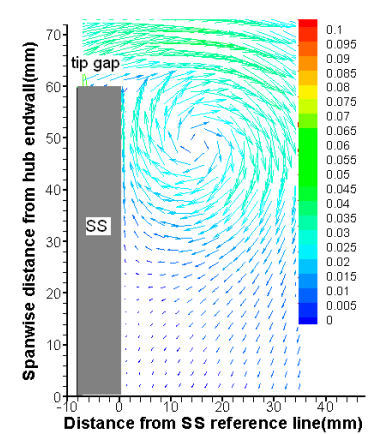

(b)

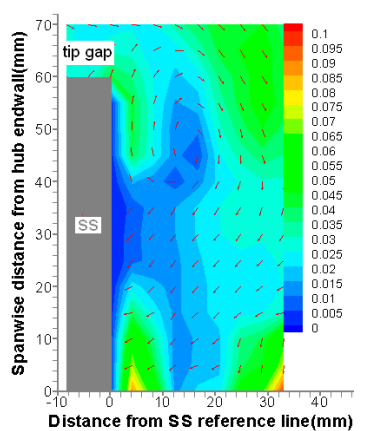

(c)

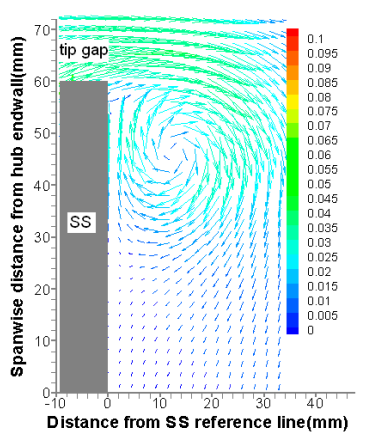

(d)

Figure 5. Mean velocity distributions on cuts 1 and 2 (m/s): (a) Cut 1-experiment; (b) Cut 1-simulation; (c) Cut 2-experiment; (d) Cut 2-simulation.

\section{Discussion of Results for BSSS, BSSM, and BRSS}

As shown in Figure 1, the experimental methods used in the previous investigations of the tip leakage flow can be classified into three groups, according to the movement type of the blade and the shroud: (1) BSSS (the blade is stationary and the shroud is also stationary); (2) BSSM (the blade is stationary but the shroud moves, to realize the same relative rotation); (3) BRSS (the blade has a rotation speed and the shroud is stationary). Using the validated numerical strategy, the characteristics of mainstream, cooling air and tip leakage are simulated with the real structure and size of a GE- ${ }^{3}$ rotor in the three cases. The simulations are conducted at ambient temperature and pressure. The tip gap is $0.4 \mathrm{~mm}(1.2 \%$ of the blade chord), and the radius of the shroud circle $\mathrm{r}$ is $300 \mathrm{~mm}$. The mainstream and cooling air are deemed to be an ideal gas. The mean velocity of the mainstream at the inlet is $60 \mathrm{~m} / \mathrm{s}$, and the corresponding Reynolds number is $3.89 \times 10^{5}$, based on the blade chord.

\subsection{Comparisons of the Tip Leakage Flow Field in Three Cases}

Figure 6 exhibits the tip leakage flow field on the cross sections of cuts 3 and 4 in the three cases at a blowing ratio of $B R=1.5$. The length and arrow of the vectors in Figure 6 represent the value and direction of local velocities, respectively. In the BRSS case, the rotation speed of the blade $\omega$ is 
$2500 \mathrm{r} / \mathrm{min}$, and in the BSSM case, a corresponding reverse velocity of the shroud is set. For the convenience of comparing the tip leakage behavior, the blade is regarded as the reference system.

As shown in Figure 6, both the trajectories of cooling air and tip leakage are clearly presented in the three cases, and the fluid velocities are relatively higher in the exits of the film hole and gap. BRSS corresponds to the real operating condition of the turbine rotor, and therefore can be regarded as the reference case here. Comparing Figure $6 a-c$, the differences between the velocity distributions of tip leakage and cooling air can be obviously observed. In Figure $6 c$, there are two vortices, one is counter-clockwise and the other is clockwise, but in Figure 6a,b, no complete vortices can be observed. In addition, the averaged velocities passing through cut 4 in the BSSS case are higher than those in the BRSS and BSSM cases.
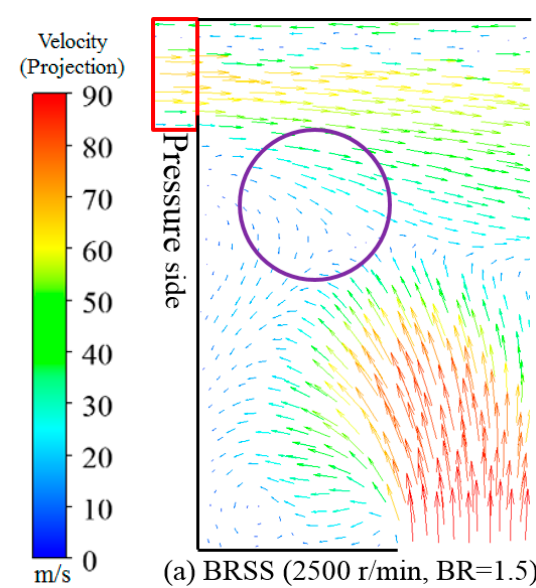

(a) BRSS (2500 r/min, $\mathrm{BR}=1.5)$

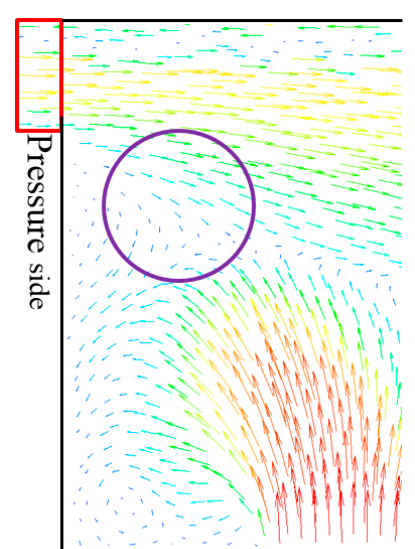

(b) $\operatorname{BSSM}(2500 \mathrm{r} / \mathrm{min}, \mathrm{BR}=1.5)$

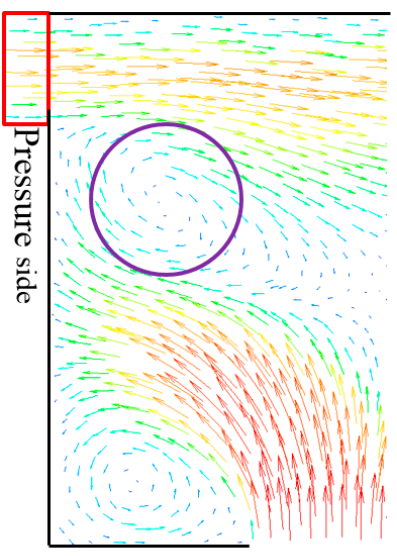

(c) BSSS (BR=1.5)

Figure 6. Velocity distributions on cuts 3 and 4 at BR = 1.5 (a) BRSS; (b) BSSM; (c) BSSS.

To clearly display the differences of tip leakage flow near the shroud region among the three cases, the velocity field in cut 4, which corresponds to the red rectangle region in Figure 6, is enlarged and presented in Figure 7. As shown in Figure 7, the tip leakage behaviors near the shroud in the BRSS and BSSM cases are identical, but both are different from that in the BSSS case. The main differences appear in the velocity field near the shroud wall, and the reason is that the blade and shroud move relatively in the BRSS and BSSM cases, while keeping stationary in the BSSS case.

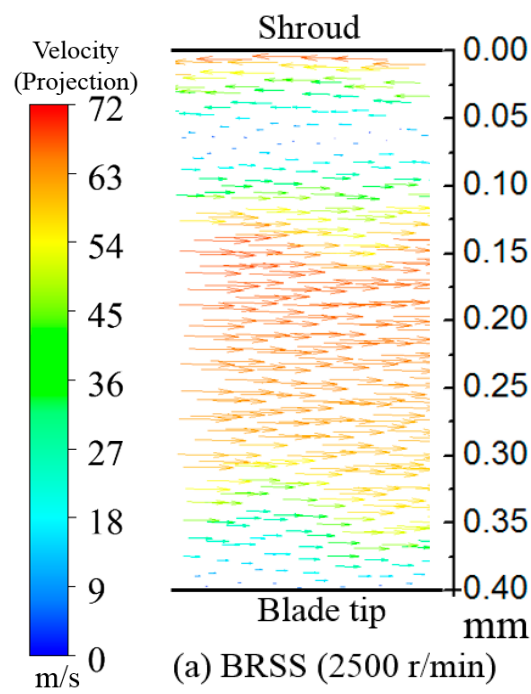

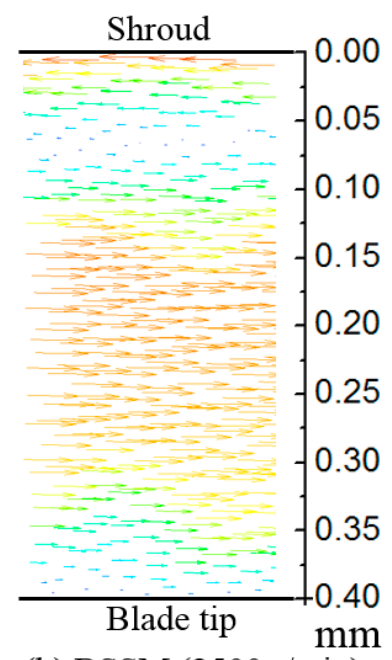

(b) $\operatorname{BSSM}(2500 \mathrm{r} / \mathrm{min})$

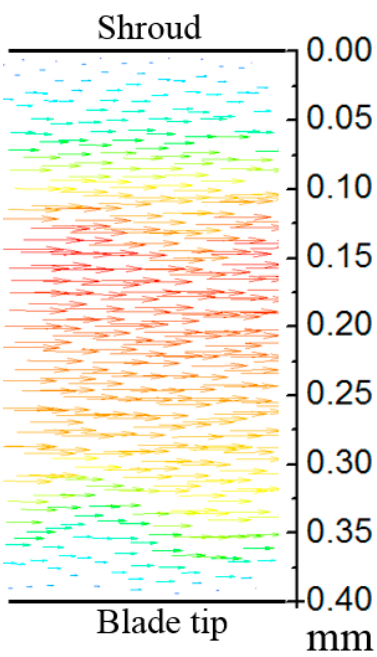

(c) BSSS

Figure 7. Velocity distributions on cut 4 at BR =1.5: (a) BRSS; (b) BSSM; (c) BSSS. 
The essential reason for the differences as discussed above is the viscous force of fluid. Figure 8 illustrates the kinetic models of the three cases. In the BSSS case, the velocities of tip leakage flow relative to the blade tip and shroud are the same, so the viscous forces at the two surfaces are nearly the same. However, the velocities relative to the blade tip and shroud are $v$ and $\omega r+v$, respectively, in the BRSS case, which results in a great difference in the viscous forces at the two surfaces. In the BSSM and BRSS cases there are similar velocity and viscous force distributions, and therefore the flow fields at the tip gap in the two cases are similar, but they are different from the BSSS case.

It is well known that the tip leakage flow results in a mainstream energy loss and an increase in the thermal load at the tip surface. In the real operating condition, the rotation rate is extremely high, so that the effect of the fluid viscous force at the tip gap cannot be ignored. This means that the errors caused by BSSS simplification cannot be eliminated. BRSS corresponds to the real operating condition of the gas turbine rotor, and BSSM can provide a similar velocity field to BRSS, in which the fluid fields obtained by the experiments can reflect the true situation. However the experimental methods of BRSS and BSSM are extremely expensive, and therefore the BSSS method is usually employed for economic reasons.

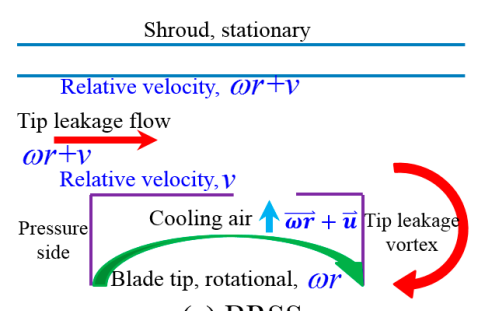

(a) BRSS

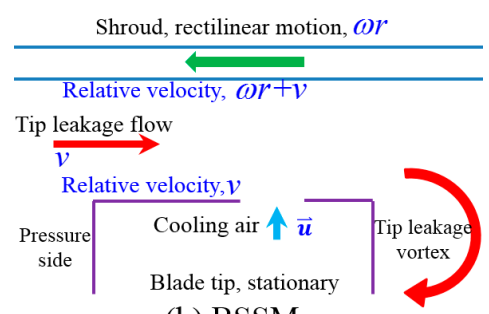

(b) BSSM

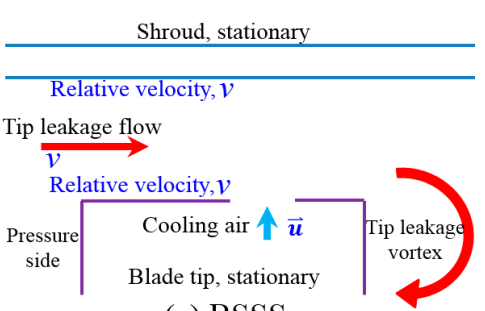

(c) BSSS

Figure 8. Comparison of the kinetic models: (a) BRSS; (b) BSSM; (c) BSSS.

\subsection{Comparisons of the Passage Flow Field in the Three Cases}

Tip leakage flow usually leads to tip leakage vortex (TLV) and passage vortex (PV), which will reduce the safety and efficiency of the gas turbine rotor. Therefore, the characteristics of TLV and PV were considered. Figure 9 illustrates the velocity distributions on cut 5 in the three cases.
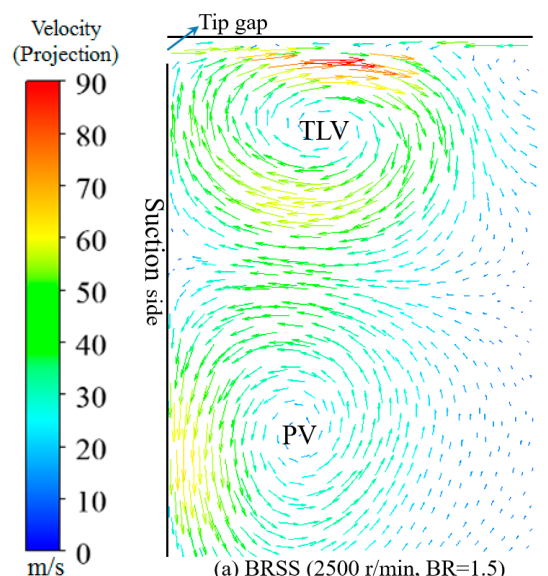

(a) BRSS $(2500 \mathrm{r} / \mathrm{min}, \mathrm{BR}=1.5)$

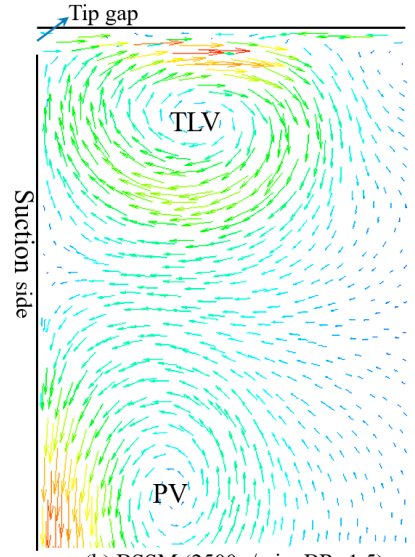

(b) $\operatorname{BSSM}(2500 \mathrm{r} / \mathrm{min}, \mathrm{BR}=1.5)$

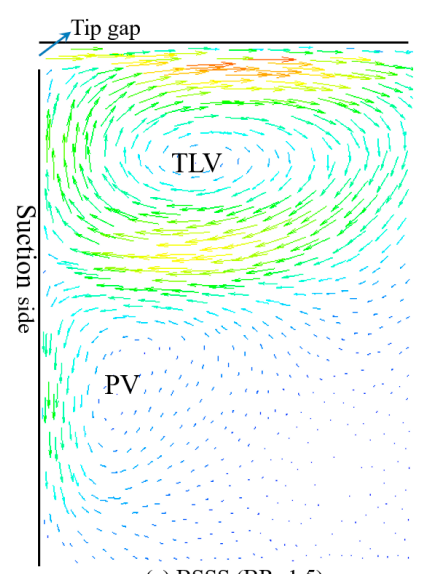

(c) $\operatorname{BSSS}(\mathrm{BR}=1.5)$

Figure 9. Velocity vectors at cut 5 at BR = 1.5: (a) BRSS; (b) BSSM; (c) BSSS.

According to the Figure 9, the shapes, sizes and locations of TLV and PV in the BSSM and BRSS cases are similar, but different from that in the BSSS case. 


\subsection{Comparisons of the Tip Leakage Mass Flow Rate in the Three Cases}

The tip leakage mass flow rate is an important factor influencing turbine efficiency. To compare quantitatively the tip leakage mass flow in the three cases, a series of simulations were carried out at the rotation speeds of 1000, 2500, $4000 \mathrm{r} / \mathrm{min}$. The numerical results of the mass flow rate and the deviations of BSSS and BSSM from BRSS are listed in Table 3. From Table 3, the following three interesting conclusions can be drawn: (1) Regardless of the rotation rate, the tip leakage mass flow rate in BSSS was higher, while the BSSM cases were lower than the BRSS case; (2) The deviations of the BSSS and BSSM cases from the BRSS case both increased when the rotation rate increased; (3) The experimental data for BSSM was better than that for BSSS.

From Table 3, one can find that the rotation rate is a critical factor, which may lead to different deviations of the tip leakage mass flow rate in three cases. Figure 10 shows the velocity field of the tip leakage flow on cut 4 in the BRSS case at the rotation speeds 1000, 2500 and $4000 \mathrm{r} / \mathrm{min}$, and the corresponding velocities of the shroud are $31.4,78.5$ and $125.7 \mathrm{~m} / \mathrm{s}$, respectively. From this figure, it can be observed that the velocity of the tip leakage flow near the shroud increases with the rotation rate. As a consequence, the viscous force of the fluid at the tip gap increases and the boundary layer becomes thicker. Therefore the average velocity of the tip flow decreases and the tip leakage mass flow rate passing through the tip gap reduces.

Table 3. Tip leakage mass flow rates in the three simulation cases at different rotation speeds.

\begin{tabular}{ccccc}
\hline Simulation Case & $\begin{array}{c}\text { Rotation Rate } \\
(\mathbf{r} / \mathbf{m i n})\end{array}$ & Blowing Ratio & $\begin{array}{c}\text { Deviation of Tip Leakage } \\
\text { Mass Flow From BRSS }\end{array}$ & $\begin{array}{c}\text { Tip Leakage } \\
\text { Mass Flow (g/s) }\end{array}$ \\
\hline BRSS & 1000 & 1.5 & Reference & 1.0299 \\
BSSS & 0 & 1.5 & $\uparrow 0.43 \%$ & 1.0343 \\
BSSM & 1000 & 1.5 & $\downarrow 0.20 \%$ & 1.0278 \\
\hline BRSS & 2500 & 1.5 & Reference & 0.9592 \\
BSSS & 0 & 1.5 & $\uparrow 7.8 \%$ & 1.0343 \\
BSSM & 2500 & 1.5 & $\downarrow 3.4 \%$ & 0.9265 \\
\hline BRSS & 4000 & 1.5 & Reference & 0.8521 \\
BSSS & 0 & 1.5 & $\uparrow 21.4 \%$ & 1.0343 \\
BSSM & 4000 & 1.5 & $\downarrow 8.6 \%$ & 0.7787 \\
\hline
\end{tabular}

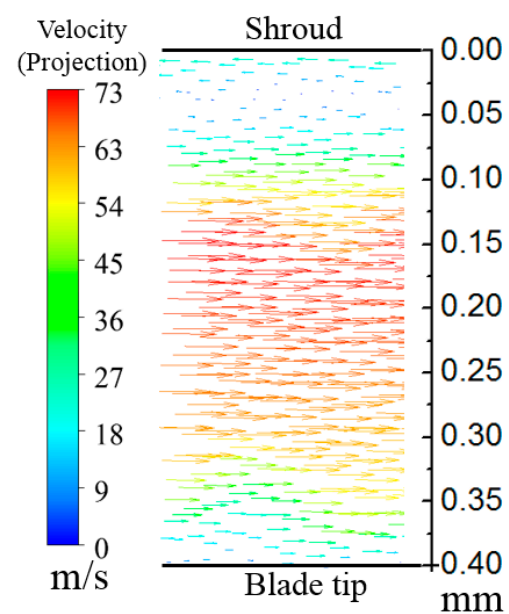

(a) BRSS (1000 r/min)

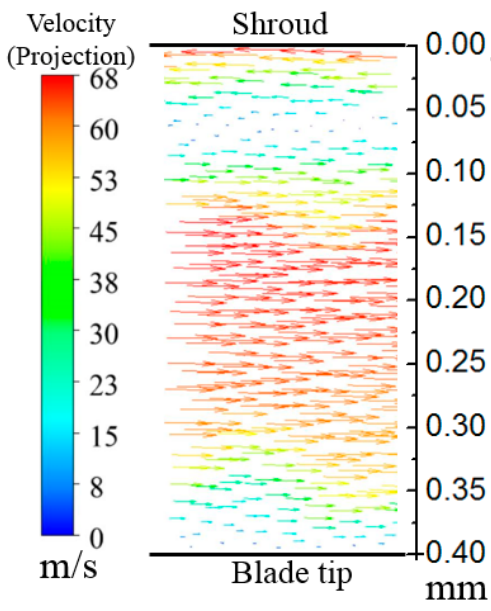

(b) BRSS (2500 r/min)

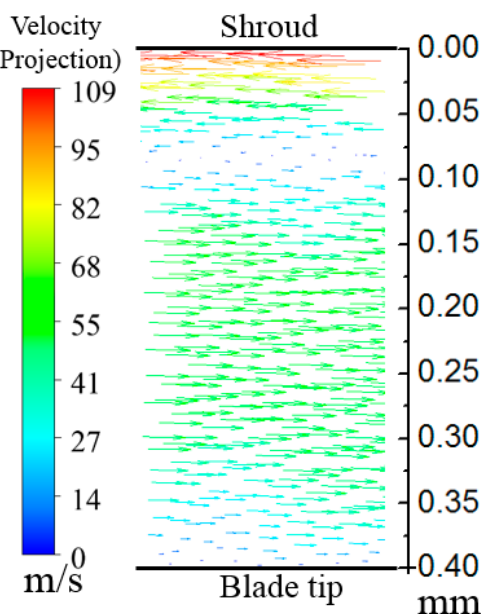

(c) BRSS (4000 r/min)

Figure 10. Velocity vectors on cut 4 at BR $=1.5$ in BRSS case: (a) $1000 \mathrm{r} / \mathrm{min}$; (b) $2500 \mathrm{r} / \mathrm{min}$; (c) $4000 \mathrm{r} / \mathrm{min}$. 


\subsection{Revision of the BSSS Case via the Shroud Wall Viscous Shear Stress}

Although the BSSS experiment method has been widely used due to its easy, economical and effective performance, the velocities of the tip leakage flow in the BRSS and BSSS cases are different, and the reason is that the viscous shear stresses at the shroud wall are not identical. To reduce the deviation of the BSSS experiment from the BRSS experiment, a simple method is proposed here, which is based on correcting the shroud wall viscosity in the BSSS case. When we add a parameter of equivalent sand-grain roughness of $h_{s}=0.04 \mathrm{~mm}$ into the shroud surface of BSSS, we can obtain the velocity vectors maps on cuts 3,4 and 5 , which are similar to the case for BRSS, as shown in Figure 11. Of course, the roughness parameter is adjusted and dependent on rotation speed. However, the velocity of the tip leakage flow is not the same value at the tip gap, and therefore the viscosity at the shroud is different at different locations, so how to choose a suitable shroud surface roughness deserves to be further studied.

Comparing Figure 11a-c with Figures $6 a, 7 a$ and $9 a$, it can be found that the BSSS case with a rough shroud surface is very similar to the BRSS case, including the tip leakage flow in the gap, and the mainstream, TLV and PV in the passage. The tip leakage mass flow rate decreased from $1.0343 \mathrm{~g} / \mathrm{s}$ to $0.9939 \mathrm{~g} / \mathrm{s}$ after revision, and the deviation of the BSSS case from the BRSS case is greatly reduced. Therefore, the surface roughness is an important parameter, when the BSSS experiment method is used to study the real gas turbine system, and a shroud with the appropriate roughness can improve the accuracy of experimental results significantly.
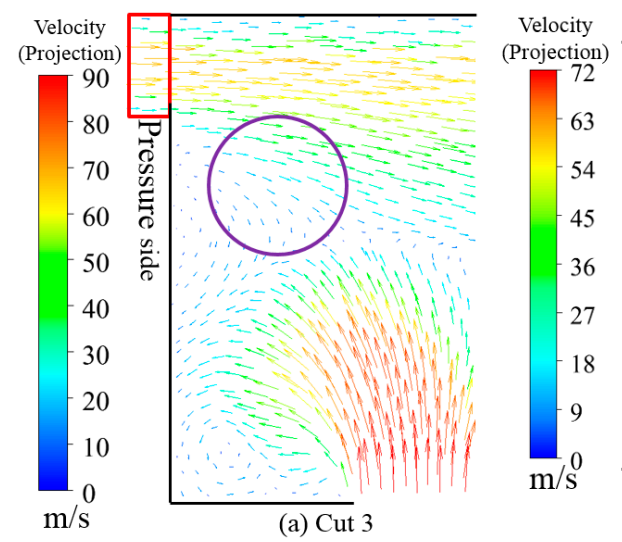

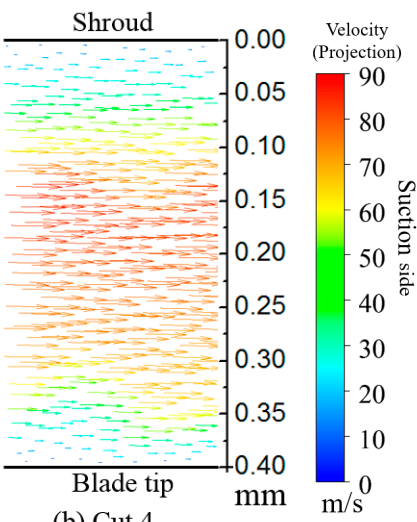

(b) Cut 4

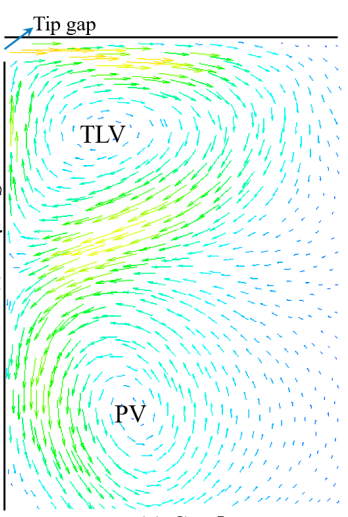

(c) Cut 5

Figure 11. Velocity vectors at the BR of 1.5 and rotation rate of $2500 \mathrm{r} / \mathrm{min}$ in BSSS case after modification using $h_{s}=0.04 \mathrm{~mm}$ : (a) Cut 3; (b) Cut 4 ; (c) Cut 5 .

\section{Conclusions}

An experimental and numerical investigation was carried out to analyze the differences between three experimental methods used in previous studies of the tip leakage flow (BSSS, BSSM and BRSS). The blade geometry used in this work had the same profile as a GE- $\mathrm{E}^{3}$ rotor, and a novel squealer tip film cooling design. Velocity fields in tip leakage flow were measured by the PIV technique, and the experimental data were used to validate the numerical strategy. Using the validated numerical strategy, the differences between the three tip models (BSSS, BSSM and BRSS) were exhibited and discussed. Through the discussions, the following usable conclusions were drawn:

1. The BSSM experiment method is closer to the real status, BRSS, including the tip leakage flow in the gap and the tip leakage vortex and the vortex generated in the passage. Therefore, the BSSM method is the more reasonable method.

2. Some evident differences between the BSSS and BRSS cases were exhibited and analyzed in this paper. The main reason causing these differences is viscous force arising in different movement patterns. In consideration of the relative movement of the blade to the shroud, the BSSS case has 
a different velocity and viscous force to the BRSS case in the tip gap. Therefore, the flow fields at the tip gap in the BRSS and BSSS cases are different.

3. In the BSSS case, the tip leakage mass flow rate is much higher than that in the real case, BRSS. The deviation proportion is $7.8 \%$, at a rotation rate of $2500 \mathrm{r} / \mathrm{min}$ and $\mathrm{BR}=1.5$, and this proportion increased significantly when the rotation rate increased.

4. A method based on correcting the wall viscous shear stress via shroud wall roughness was proposed to revise the experimental data obtained in the BSSS case, so that the difference of the BSSS case from the real case, BRSS, is reduced and the results obtained are more accurate.

Acknowledgments: The authors gratefully acknowledge the Natural Science Foundation of China (contract no. 51376168) and the 1st Group of Chinese Aviation Company for their support for this investigation.

Author Contributions: Xiaochun Wang, Fei He and Hong Zhang conceived, designed and performed the experiments. Xiaochun Wang and Hong Zhang performed the simulations. Xiaochun Wang, Fei He and Jianhua Wang analyzed the data. Jianhua Wang wrote the paper.

Conflicts of Interest: The authors declare no conflict of interest. The founding sponsors had no role in the design of the study; in the collection, analysis, or interpretation of data; in the writing of the manuscript, and in the decision to publish the results.

\section{References}

1. Bunker, R.S. A Review of Turbine Blade Tip Heat Transfer. Ann. N. Y. Acad. Sci. 2001, 934, 64-79. [CrossRef] [PubMed]

2. Kwak, J.S.; Ahn, J.; Han, J.-C.; Lee, C.P.; Bunker, R.S.; Boyle, R.; Gaugler, R. Heat transfer coefficients on the squealer tip and near-tip regions of a gas turbine blade with single or double squealer. J. Turbomach. 2003, 125, 778. [CrossRef]

3. Azad, G.S.; Han, J.C.; Boyle, R.J. Heat Transfer and Flow on the Squealer Tip of a Gas Turbine Blade. J. Turbomach. 2000, 122, 725-732. [CrossRef]

4. Thorpe, S.J.; Yoshino, S.; Thomas, G.A.; Ainsworth, R.W.; Harvey, N.W. Blade-tip heat transfer in a transonic turbine. Proc. Inst. Mech. Eng. Part A J. Power Energy 2006, 219, 421-430. [CrossRef]

5. Nasir, H.; Ekkad, S.V.; Kontrovitz, D.M.; Bunker, R.S.; Prakash, C. Effect of tip gap and squealer geometry on detailed heat transfer measurements over a high pressure turbine rotor blade tip. J. Turbomach. 2004, 126, 221. [CrossRef]

6. Kwak, J.S.; Han, J.C. Heat Transfer Coefficient and Film-Cooling Effectiveness on the Squealer Tip of a Gas Turbine Blade. In Proceedings of the ASME Tubro Expo 2002, Amsterdam, The Netherlands, 3-6 June 2002; GT2002-30555.

7. Yaras, M.I.; Sjolander, S.A. Effects of Simulated Rotation on Tip Leakage in a Planar Cascade of Turbine Blades: Part I-Tip Gap Flow. J. Turbomach. 1992, 114, 652-659. [CrossRef]

8. Yaras, M.I.; Sjolander, S.A.; Kind, R.J. Effects of Simulated Rotation on Tip Leakage in a Planar Cascade of Turbine Blades: Part II-Downstream Flow Field and Blade Loading. J. Turbomach. 1992, 114, 660-667. [CrossRef]

9. Palafox, P.; Oldfield, M.L.G.; LaGraff, J.E.; Jones, T.V. PIV Maps of Tip Leakage and Secondary Flow Fields on a Low Speed Turbine Blade Cascade with Moving Endwall. In Proceedings of the ASME Tubro Expo 2005, Reno-Tahoe, NV, USA, 6-9 June 2005; GT2005-68189.

10. Palafox, P.; Oldfield, M.L.G.; Ireland, P.T.; Jones, T.V.; LaGraff, J.E. Blade Tip Heat Transfer and Aerodynamics in a Large Scale Turbine Cascade with Moving Endwall. In Proceedings of the ASME Tubro Expo 2006, Barcelona, Spain, 8-11 May 2006; GT2006-90425.

11. Srinivasan, V.; Goldstein, R.J. Effect of Endwall Motion on Blade Tip Heat Transfer. J. Turbomach. 2003, 125, 267-273. [CrossRef]

12. Rhee, D.-H.; Cho, H.H. Local heat/mass transfer characteristics on a rotating blade with flat tip in low-speed annular cascade-Part I: Near-tip surface. J. Turbomach. 2006, 128, 96. [CrossRef]

13. Rhee, D.-H.; Cho, H.H. Local heat/mass transfer characteristics on a rotating blade with flat tip in a low-speed annular cascade-Part II: Tip and shroud. J. Turbomach. 2006, 128, 110. [CrossRef] 
14. Tamunobere, O.; Drewes, C.; Acharya, S.; Nakamata, C. Heat transfer to an actively cooled shroud with blade rotation. J. Therm. Sci. Eng. Appl. 2015, 7, 041020. [CrossRef]

15. Tamunobere, O.; Acharya, S. Turbine blade tip film cooling with blade rotation-Part I: Tip and pressure side coolant injection. J. Turbomach. 2016, 138, 091002. [CrossRef]

16. Tamunobere, O.; Acharya, S. Turbine blade tip cooling with blade rotation-Part II: Shroud coolant injection. J. Turbomach. 2016, 138, 091003. [CrossRef]

17. Lavagnoli, S.; De Maesschalck, C.; Andreoli, V. Design considerations for tip clearance control and measurement on a turbine rainbow rotor with multiple blade tip geometries. J. Eng. Gas Turbines Power 2016, 139, 042603. [CrossRef]

18. Yang, D.; Yu, X.; Feng, Z. Investigation of leakage flow and heat transfer in a gas turbine blade tip with emphasis on the effect of rotation. J. Turbomach. 2010, 132, 041010. [CrossRef]

19. Acharya, S.; Moreaux, L. Numerical study of the flow past a turbine blade tip: Effect of relative motion between blade and shroud. J. Turbomach. 2013, 136, 031015. [CrossRef]

20. Krishnababu, S.K.; Dawes, W.N.; Hodson, H.P.; Lock, G.D.; Hannis, J.; Whitney, C. Aero Thermal Investigation of Tip Leakage Flow in Axial Flow Turbines Part II-Effect of Relative Casing Motion. In Proceedings of the ASME Tubro Expo 2007, Montreal, QC, Canada, 14-17 May 2007; GT2007-27957.

21. Zhou, C.; Hodson, H.; Tibbott, I.; Stokes, M. Effects of winglet geometry on the aerodynamic performance of tip leakage flow in a turbine cascade. J. Turbomach. 2013, 135, 051009. [CrossRef]

22. Lee, W.S.; Kim, D.H.; Park, J.S.; Kwak, J.S.; Chung, J.T. Effect of triangular grooved tip on blade tip region heat transfer. J. Thermophys. Heat Transf. 2014, 28, 226-235. [CrossRef]

23. Wang, J.; Sundén, B.; Zeng, M.; Wang, Q. Film cooling effects on the tip flow characteristics of a gas turbine blade. Propuls. Power Res. 2015, 4, 9-22. [CrossRef]

24. Kang, D.B.; Lee, S.W. Effects of squealer rim height on heat/mass transfer on the floor of cavity squealer tip in a high turning turbine blade cascade. Int. J. Heat Mass Transf. 2016, 99, 283-292. [CrossRef]

25. Zhong, F.; Zhou, C.; Ma, H.; Zhang, Q. Heat transfer of winglet tips in a transonic turbine cascade. J. Eng. Gas Turbines Power 2016, 139, 012605. [CrossRef]

26. Hahn, M.; Drikakis, D. Implicit Large-Eddy Simulation of Swept-Wing Flow Using High-Resolution Methods. AIAA J. 2009, 47, 618-629. [CrossRef]

27. Hahn, M.; Drikakis, D. Assessment of Large-Eddy Simulation of Internal Separated Flow. J. Fluids Eng. 2009, 131, 071201. [CrossRef]

28. Leschziner, M.A.; Drikakis, D. Turbulence modelling and turbulent-flow computation in aeronautics. Aeronaut. J. 2002, 106, 349-384.

29. Yang, H.T.; Acharya, S.; Ekkad, S.V.; Prakash, C.; Bunker, R. Flow and Heat Transfer Predictions for a Flat-tip Turbine Blade. In Proceedings of the ASME Tubro Expo 2002, Amsterdam, The Netherlands, 3-6 June 2002; GT2002-30190.

(C) 2017 by the authors. Licensee MDPI, Basel, Switzerland. This article is an open access article distributed under the terms and conditions of the Creative Commons Attribution (CC BY) license (http://creativecommons.org/licenses/by/4.0/). 